Dr. Shiri Shinan-Altman, Ph.D., Louis and Gabi Weisfeld School of Social Work, Bar Ilan University, Ramat-Gan, Israel. Corresponding author: shiri. altman@biu.ac.il

Dr. Inbar Levkovich, Ph.D., Faculty of Graduate Studies, Oranim Academic College of Education, Israel.

Dr. Amit Porat, M.D., Department of Family Medicine, The Ruth \& Bruce Rappaport Faculty of Medicine, Technion-Israel Institute of Technology, Clalit Health Services, Haifa and Western Galilee District, Israel.

Dr. Mordechai Alperin, M.D, Department of Family Medicine, The Ruth \& Bruce Rappaport Faculty of Medicine, Technion-Israel Institute of Technology, Clalit Health Services, Haifa and Western Galilee District, Israel.

\title{
THE IMPACT OF THE COVID-19 EPIDEMIC ON OLDER ADULTS' EXPERIENCES WITH HEALTHCARE SERVICES
}

\section{Abstract}

Objective: Maintaining healthcare services consumption as needed during a pandemic crisis is important, in general, and specifically for older adults. Therefore, the current study aimed to examine factors associated with experiences related to healthcare services among Israeli older adults during the COVID-19 pandemic.

Methods: A cross-sectional online survey was conducted among 261 older adults (aged 60+). Participants completed measures of perceived health status, COVID-19 perceived risk, knowledge about COVID-19, depression, coping resources, experiences with healthcare services (contact with a family physician, fear of contracting the virus via direct contact with the healthcare system). Two multiple regressions were calculated for the following dependent variables: contact with a family physician during COVID-19, and fear of contracting the virus via direct contact with the healthcare system during COVID-19.

Results: Older adults' contact with a family physician during COVID-19 was lower than their contact prior to the pandemic. A higher extent of contact with a family physician during COVID-19 was related to higher knowledge about COVID-19. In addition, a higher extent of fear of contracting the virus via direct contact with the health system during COVID-19 was related to lower years of education, higher COVID-19 perceived risk, and lower knowledge about COVID-19.

Conclusions: The results could provide public health policymakers with a more complete picture of the impact of the COVID-19 crisis among older adults. This study highlights the characteristics and factors that encourage/discourage older 
adults from seeking healthcare services during a virus outbreak, such as the COVID-19 pandemic.

Key words: coping resources, COVID-19, depression, experiences with healthcare services, knowledge about COVID-19, older adults, perceived risk

\section{Introduction}

According to different studies conducted around the globe, coronavirus disease (COVID-19) is highly linked with reduced health care utilization (Lazzerini et al., 2020; Shinan-Altman et al., 2020). For example, in the United States there was a decrease of $38 \%$ in severe heart attack patients treated in nine main medical centres (Garcia et al., 2020); in Italy, a decrease of $73 \%$ to $88 \%$ was reported in paediatric emergency department visits (Lazzerini et al., 2020); and in Israel, $88 \%$ of breast cancer patients cancelled an appointment with the healthcare services due to COVID-19 (Shinan-Altman et al., 2020). Another study conducted in Israel found that within two months of the COVID-19 outbreak, the average number of visits to the emergency department dropped by $30.2 \%$ and the hospital occupancy by $29.2 \%$, compared to the same period the year before (Basis et al., 2020). Reduced or delayed healthcare utilization during a pandemic can have detrimental health consequences (Shinan-Altman et al., 2020). This is especially true for older adults (aged 60+), as reduced healthcare utilization can affect them both physically and emotionally (Morrow-Howell et al., 2020). Indeed, the COVID-19 crisis has instilled fear and depression in older people, particularly as a result of the reports and evidence showing that this population has higher fatality rates and higher susceptibility to contract the virus (Radwan et al., 2020). During periods of epidemic outbreaks and other health crises, older adults experience difficult challenges (Morrow-Howell et al., 2020). As older adults often turn to their family physician to address medical and mental concerns (Elliott et al., 2018), the role of family physicians becomes even more significant during such times.

Continuity of care and treatment is one of the central principles of family medicine (Adar et al., 2017; Jahromi et al., 2017), and it is especially important in times of pandemic. In the primary care framework, continuity of care is most often viewed as the physician-patient relationship that extends beyond episodes of illness (Elliott et al., 2018). Nevertheless, during the COVID-19 outbreak, it seems that one of the main risks for older adults has been the inability to receive routine medical care in the community. This situation may be explained in various ways: First, older adults were advised to adopt isolation measures and restrict their movement, in order to protect themselves from infection (Israeli Ministry of Health; MoH, 2020). Second, the healthcare system has narrowed its focus to managing COVID-19 patients, meaning other appointments and procedures were delayed or cancelled (Morrow-Howell et al., 2020). Third, travel restrictions have 
made it difficult for some older adults to reach health services facilities. Finally, the fear of contracting the virus while visiting health services has led to the delay and cancellation of appointments in the health system (World Health Organization, 2020). Consequently, older adults might not be consuming the health services they need (Morrow-Howell et al., 2020) during this time. In order to shed light on this phenomenon, the current study aimed to examine factors associated with Israeli older adults' experiences with the healthcare services during the COVID-19 pandemic.

Previous studies in the area of health services consumption revealed a variety of different cognitive and emotional variables as well as coping resources that may explain health services utilization. Cognitive variables include perceptions (e.g., perceived health status, perceived risk of contracting a disease/virus) and knowledge about a disease/virus (Rastogi, Madhok and Kipperman, 2013; Shinan-Altman et al., 2020). Regarding emotional variables, in this study we concentrated on depression, as in Israel - similar to other countries (e.g., Castro-Costa et al., 2007; Li et al., 2014) - one of every four older adults suffer from significant depressive symptoms (Bentur and Heymann, 2020). In addition, higher depressive symptoms were reported among older adults during the COVID-19 outbreak (Santini et al., 2020). Furthermore, higher depressive symptoms were found to be associated with increased outpatient resource utilization and more visits to family physicians
(Bentur and Heymann, 2020). Finally, in the current study coping resources included optimism (an internal resource) and social support (an external resource), as these coping resources were found to be significantly associated with health services consumption (DeViva et al., 2020; Wang et al., 2020).

Both cognitive and emotional factors, together with coping resources, have significant bearing on the way individuals tend to deal with health threats (Zhang et al., 2014). However, studies regarding these concepts during the COVID-19 period are only now beginning to emerge, and to the best of our knowledge, no study has yet examined these factors among older adults during a pandemic. Therefore, the goal of the current study was to examine determinants of older adults' healthcare experiences during COVID-19. Gaining deeper insight into the mechanisms related to health services utilization among older adults may help improve their physical and mental health indicators, especially during a virus outbreak.

\section{Method}

Procedure: The study was conducted with "Clalit Health Services" (Haifa and Western Galilee District), the largest non-profit health maintenance organization (HMO) in Israel. Data were collected between June and July 2020. This period of time reflects the subsiding of the first wave of COVID-19 in Israel. During this time, many individuals were requested to take a leave of absence or worked from 
home. A new routine was established in Israel, including the mandatory wearing of face masks and strict social distancing measures.

A total of 261 older adults participated in the study. Inclusion criteria: (a) insured by "Clalit Health Services", (b) over the age of 60, and (c) a Hebrew speaker. Exclusion criterion: cognitive impairment. Potential participants who met the inclusion criteria were identified by managers from eight clinics - half were located in urban areas and half in rural areas. Potential participants' contact details were forwarded to six family medicine interns, who contacted them and explained the characteristics and importance of the study. Patients who agreed to participate were interviewed by the family medicine interns via telephone, using a structured pre-tested questionnaire. Of the 278 patients who were approached, 261 agreed to participate (a $94 \%$ response rate). A total of 17 patients refused to participate due to a lack of interest. The study was approved by the Helsinki committee of "Clalit Health Services" (approval number 0060-20-COM2).

\section{Measures}

\section{Dependent variables}

Healthcare experiences during COVID-19 was measured using two dimensions:

1. Contact with a family physician during COVID-19 was measured using a 2 -item scale. One item refers to the degree of contact with a family physician prior to the COVID-19 outbreak; an additional item refers to the degree of contact with a family physician during the COVID-19 outbreak. Participants were asked to indicate the extent of their contact with their family physician on a 5-point Likert scale ranging from 1 (not at all) to 5 (very often) (e.g., "To what extent were you in contact with a family physician prior to the outbreak of COVID-19?"). A higher score indicates a higher frequency of contact with a family physician.

2. Fear of contracting COVID-19 via direct contact with the health system was measured using a single item. Participants were asked to indicate the extent of fear of contracting COVID-19 they experienced when interacting directly with the health system on a 5-point Likert scale ranging from 1 (not at all) to 5 (very much).

\section{Independent variables}

COVID-19 perceived risk was assessed based on previous studies conducted among the lay public (e.g., Shinan-Altman and Levkovich, 2020) using a single item: "What do you think is the likelihood that you will contract COVID-19?" Answers were rated on a 5-point Likert-type scale, ranging from 1 (negligible) to 5 (very high).

Knowledge about COVID-19 was measured using a 6-item COVID-19 knowledge test. The scale's validity was reached by expert validity. Answers were rated on a 5-point Likert-type scale, ranging from 1 (I don't know anything at all) 
to 5 (I know a lot). A composite index of the average of all items was created, with a higher score indicating higher levels of knowledge about COVID-19 (Cronbach's $\alpha=0.70$ ).

Depression was measured using the Symptoms of Depression Questionnaire (Centre for Epidemiological Studies Depression, CESD-10; Andresen et al., 1994). This instrument is comprised of 10 items and is a shortened version of a 20-item instrument (Radloff, 1977). The items were subcategorized as positive (e.g., "generally satisfied") and negative (e.g., "feeling tired or low in energy") (Irwin et al., 1999; Kohout et al., 1993). Participants were asked to rate the intensity of their experiences during the last week on a 4-point Likert-type scale ranging from 1 (never) to 4 (to a great extent). After reversing the negative statements, the mean score was calculated, with higher scores reflecting a higher level of depression (Cronbach's $\alpha=0.75$ ).

Optimism was measured using the Life Orientation Test (LOT-R; Scheier et al., 1994). This instrument is a six-item scale, with three items worded as positive statements (e.g., "In uncertain times, I usually expect the best") and the other three as negative statements (e.g., "If something can go wrong for me, it will"), which reflect a patient's expectations regarding the future. Participants were asked to indicate the extent to which they agreed or disagreed with each item on a 5-point Likert scale ranging from 1 (completely agree) to 5 (completely disagree).After reversing the negative statements, a mean score was calculated, with higher scores indicating a higher level of optimism (Cronbach's $\alpha=0.61$ ).

Social support was measured using the Multidimensional Scale of Perceived Social Support (Zimet et al., 1988). Using this 12-item scale, participants were asked to indicate the extent to which they agreed or disagreed with each item on a 5-point Likert scale ranging from 1 (strongly disagree) to 5 (strongly agree) (e.g., "My family really tries to help me"). The mean score was calculated; a high score indicated greater levels of perceived social support (Cronbach's $\alpha=0.81$ ).

Socio-demographic and health characteristics included gender, age, years of education, marital status, number of children with whom participants live, and sources of help during COVID-19. Regarding health characteristics, the study relied on participants' self-reports regarding: perceived health status ( $1=$ excellent, $5=$ very bad) and other types of chronic diseases (such as hypertension, diabetes, coronary heart disease, cancer, lung disease, diabetes, hypertension, other).

\section{Statistical analyses}

Data were analysed using SPSS ver. 25. Descriptive statistics were used to describe participants' demographic characteristics and research variables. Pearson correlations were calculated to assess the associations between the study and background variables.

None of the socio-demographic and health characteristics were found to be sig- 
nificantly related to contact with a family physician during COVID-19. As for fear of contracting the virus via direct contact with the health system during COVID-19, this variable was significantly and positively associated with gender $(r=0.15$, $p<0.05)$, and significantly and negatively associated with years of education ( $\mathrm{r}$ $=-0.18, p<0.01$ ).

Two multiple regressions were calculated for the dependent variables: contact with a family physician during COVID-19 and fear of contracting the virus via di- with the health system during COVID-19. All continuous variables were standardized. To assess for multicollinearity, we examined correlations between the covariates and found no strong associations (i.e., $r<0.55$ ).

\section{Results}

Description of the sample: The study included 261 older adults. As shown in Table 1, the majority of the participants were female, and the mean age was 71.82

Table 1. Participants' demographic characteristics $(n=261)$

\begin{tabular}{ll} 
Socio-demographic characteristics & \\
Gender (\%) & $119(46.7)$ \\
Male & $136(53.3)$ \\
Female & $71.82(6.91), 60-95$ \\
Mean age (SD), range & \\
Marital status (\%) & $189(74.1)$ \\
Married & $66(25.9)$ \\
Single & $3.04(1.28), 0-10$ \\
Mean number of children (SD), range & $13.10(3.11), 6-24$ \\
Mean number of years of education (SD), range & \\
Living with (\%) & $46(18.5)$ \\
Alone & $176(70.7)$ \\
Intimate partner & $26(10.4)$ \\
Family member & $1(0.4)$ \\
Formal caregiver & \\
Sources of help during COVID-19 (\%) & \\
More information about COVID-19 & $55(23.5)$ \\
Professional support (e.g., social worker, psychologist) & $18(7.7)$ \\
Family physician support & $60(25.7)$ \\
Family and friends support & $56(23.9)$ \\
Other & $45(19.2)$ \\
\hline SD Standard Deviation) &
\end{tabular}

$(\mathrm{SD}=$ Standard Deviation)

rect contact with the health system during COVID-19. Following the bivariate analysis, gender and years of education were included in the regression for fear of contracting the virus via direct contact years, with a range of 60 to 95 . The majority were married with an average of three children. The participants had an average of 13 years of education, most of them lived with an intimate partner, and about 
$25.7 \%$ perceived their family physician as a source of help during COVID-19.

Regarding participants' health characteristics, about $79.5 \%$ perceived their health status as moderate to poor; about $83 \%$ had a chronic disease, with hypertension being reported as the leading chronic disease [other chronic diseases were diabetes $(8.8 \%)$, coronary heart disease $(10.2 \%)$, cancer $(11.6 \%)$, lung disease $(5.6 \%)$, both diabetes and hypertension (19\%), and other chronic diseases (15.6)].

Descriptive statistics and correlations among study variables: As can be seen in Table 2, the mean score for contact with a family physician during COVID-19 was 3.07 ( $\mathrm{SD}=1.17$, range $1-5)$, meaning that the contact with a family physician score was relatively high during the COVID-19 outbreak. In addition, the mean score for fear of contracting the virus via direct contact with the health system during the COVID-19 outbreak was relatively high. Furthermore, the mean scores for perceived health status, COVID-19 perceived risk, and knowledge about COVID-19 were relatively high, while the mean score for depression was relatively low. The mean score for social support was relatively high.

Contact with a family physician: A paired-sample $t$-test was conducted to evaluate the changes in contact with a family physician before and during the COVID-19 outbreak. A statistically significant decrease was found in being in contact with a family physician before the COVID-19 outbreak (Mean=3.29,

Table 2. Correlates, Means, SDs, and ranges of study variables $(n=261)$

\section{Variables}

1. Contact with a family physician

2. Fear of contracting the virus via direct contact with the health system

3. Perceived health status

$0.14 *-$

4. COVID-19 perceived risk $0.15^{*}$

5. Knowledge about COVID-19

6. Depression

7. Optimism

8. Social support

Mean

SD

Possible range

Actual range

12

2

3

4

5

6

7 8

\section{$\left({ }^{*} p<0.05 ;{ }^{* *} p<0.01 ; S D=\right.$ Standard Deviation $)$}


$\mathrm{SD}=1.11)$ and during the COVID-19 outbreak $($ Mean=3.07, $\mathrm{SD}=1.17), \mathrm{t}(157)=$ $3.78, p<.001$ (Pair 2).

Associations among the study variables: As can be observed in Table 2, the associations among the study's variables show that contact with a family physician was significantly and positively associated with perceived health status, COVID-19 perceived risk, knowledge about COVID-19, and depression. In other words, the more participants perceived their health status as moderate-poor, perceived themselves to be at a higher risk to contract COVID-19, had more knowledge about COVID-19, and had higher depression levels; the more they were in contact with mism; the more they feared contracting the virus via direct contact with the health system. In addition, perceived health status was significantly and positively associated with COVID-19 perceived risk and depression, and negatively associated with optimism and social support. Positive significant associations were found linking COVID-19 perceived risk with knowledge about COVID-19 and depression. Negative significant associations were found between COVID-19 perceived risk and optimism. Negative significant associations were found linking depression, optimism and social support; a positive significant association was found between optimism and social support.

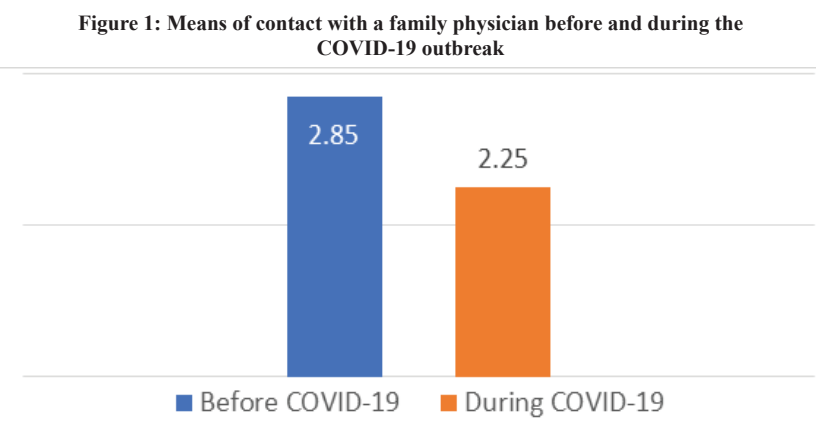

their family physician during COVID-19. Fear of contracting the virus via direct contact with the health system was significantly and positively associated with perceived health status, COVID-19 perceived risk, and depression; and negatively associated with optimism. Namely, the more participants perceived their health status as moderate-poor, perceived themselves to be at a higher risk to contract COVID-19, had higher depression, and lower opti-
Regression analysis for identifying contact with a family physician during COVID-19 correlation: The findings indicated that the full regression model was significant $(F(6,143)=2.70, p<$ $0.05)$, and explained $6 \%$ of the variance in contact with a family physician during COVID-19. As can be observed in Table 3, it appears that a higher extent of contact with a family physician during 
Table 3. Regression analysis for contact with a family physician during COVID-19 (n=261)

Dependent variable

Independent variables

Perceived health status

COVID-19 perceived risk

Knowledge about COVID-19

Depression

Optimism

Social support

Adjusted $\mathrm{R}^{2}$

$\mathrm{F}(\mathrm{df})$

Contact with a family physician during COVID-19

$\mathrm{B}$

0.12

0.06

0.17

0.20

$-0.09$

$-0.03$

$0.06 * *$

$2.70(6,143)$
Beta

0.09

0.05

$0.22 * *$

0.95

$-0.06$

$-0.03$

$(* * p<.05)$

COVID-19 was related to lower knowledge about COVID-19.

Regression analysis for identifying fear of contracting the virus via direct contact with the health system correlation: The findings indicated that the full regression model was significant $(F(8$, $136)=14.11, p<0.001)$, and explained $42 \%$ of the variance in fear of contracting the virus via direct contact with the health system. As can be observed in Table 4, it appears that a higher extent of fear of contracting the virus via direct contact with the health system during COVID-19 was related to lower years of education, higher COVID-19 perceived risk, and lower knowledge about COVID-19.

\section{Discussion}

The current study is an attempt to encourage older adults who need treatment and care to utilize healthcare services during the COVID-19 outbreak. Therefore, the goal of the study was to examine the determinants of older adults' healthcare experiences during COVID-19.

The study findings indicated that older adults' contact with a family physician was less frequent during the COVID-19 pandemic, compared to before the outbreak. This decrease in contact with a family physician among older adults during COVID-19 is worrisome for three main reasons: First, reduced healthcare use (e.g., less contact with a family physician) was found to be associated with lower health indicators (Wang et al., 2012). Second, family physicians play a significant role in older adults' struggle to cope with health-related events, as elderly people often initially turn to their family physician to address medical and mental complaints (Elliott et al., 2018). Maintaining contact with a family physician is especially important during times of a pandemic outbreak, given that many older adults experience a decline in physical and mental indicators as a result of social distancing (Tyrrell and Williams, 
Table 4. Regression analysis for fear of contracting COVID-19 via direct contact with the health system $(n=261)$

Dependent variable

Independent variables

Gender

Years of education

Perceived health status

COVID-19 Perceived risk

Knowledge about COVID-19

Depression

Optimism

Social support

Adjusted $\mathrm{R}^{2}$

$\mathrm{F}(\mathrm{df})$
Fear of contracting COVID-19 via direct

contact with the health system

$\begin{array}{ccc}\text { B } & \text { SEB } & \text { Beta } \\ 0.84 & 0.15 & 0.04 \\ -0.06 & 0.03 & -0.16^{*} \\ 0.11 & 0.09 & 0.09 \\ 0.75 & 0.10 & 0.58^{* * *} \\ 0.22 & 0.04 & 0.27^{* * *} \\ 0.23 & 0.17 & 0.10 \\ -0.01 & 0.12 & -0.01 \\ 0.14 & 0.10 & 0.09 \\ 0.42 * * * & & \\ 14.11(8,136) & & \end{array}$

$(* p<0.05, \quad * * * p<0.001)$

2020). Third, given that older adults are at a higher risk of severe illness and mortality (Shahid et al., 2020), the COVID-19 discourse has globally portrayed them as a vulnerable group. Accordingly, the "burden narrative" of the elderly population has flourished, due to an overwhelmed healthcare system that cannot provide adequate care for all (Ayalon, 2020). It is possible that older adults have decreased their contact with the healthcare system as a result of this "burden narrative".

In the current study, a higher extent of contact with a family physician was associated with higher knowledge about COVID-19. This finding is in line with a previous study conducted during the Severe Acute Respiratory Syndrome (SARS) epidemic in 2003, which found that because of limited knowledge about SARS, people were afraid to seek healthcare ser- vices (Lu, Chou and Liou, 2007). The finding that knowledge about COVID-19 is an important determinant of contact with a family physician during COVID-19 is significant for those who disseminate public health information during health crises. Accordingly, health leaders should identify vulnerable populations that may not be literate or are not connected to online social networks, and promote messages via other, more effective means, which may have a higher possibility of reaching these populations.

In this study, fear of contracting the virus via direct contact with the healthcare system during the COVID-19 outbreak was relatively high. This finding is not surprising, given that during the period of time when the current study was conducted, older adults were represented by health authorities and the media as a vulnerable 
population, at high risk of contracting COVID-19 (Ayalon, 2020). Older adults were instructed to adopt isolation measures and restrict their movements $(\mathrm{MoH}$, 2020). In addition, it was claimed that during COVID-19, the Israeli MoH had adopted a fear-based strategy (Basis et al., 2020). While there may be some value in generating fear of a health threat among people, thereby causing them to minimize their chances of contracting the disease, an extreme amount of fear might do more harm than good. It has been suggested that the Israeli MoH's strategy might have influenced citizens in an adverse manner - causing them to perceive the healthcare services facilities as places of contagion (Basis et al., 2020).

Our findings indicated that a higher extent of fear of contracting the virus via direct contact with the healthcare system during COVID-19 was related to higher COVID-19 perceived risk, lower years of education, and lower knowledge about COVID-19. These findings reinforce the importance of providing accurate information and messages to older adults, who may be less easily accessible regarding more modern (technology-based) methods, due to their education level and social isolation circumstances (So et al., 2004). These messages should convey the significance of maintaining routine contact with the healthcare system during a pandemic crisis.

This study has important implications. According to the study's results, clinical interventions targeting older adults' years of education, COVID-19 perceived risk, and knowledge about COVID-19 may encourage them to consume healthcare services during an epidemic outbreak, if needed. Health professionals can use the current findings in order to have a more complete picture about the profiles of older adults who choose to reduce contact with their family physician during the outbreak. It is also recommended that in times of pandemic outbreak the medical staff will initiate proactive phone-calls to older adults to inquire about their physical and mental well-being. These conversations are an opportunity to provide explanations about how the healthcare services are prepared to treat older adults during the epidemic. In parallel, health services should be prepared to provide the necessary care and treatment for older adults, while decreasing any possibility of them contracting the virus.

Although this preliminary study is limited by its cross-sectional and self-report design, which may affect its generalizability; and regardless of the facts that participants' medical history was not assessed, and that all participants were from the same HMO, the current study provides pioneering insight into potential negative health outcomes linked with the COVID-19 pandemic among older adults. Furthermore, this study emphasizes the qualities and factors that encourage/discourage older adults from utilizing healthcare services during a virus outbreak, such as the COVID-19 pandemic.

It is suggested to conduct future longitudinal studies, which will examine medical indicators and other psychosocial 
factors that may be related to this population's experiences with the healthcare services among larger samples of older adults and over longer time periods. These studies will enable the creation of tailored intervention programs in the eventuality of a recurrence of another health pandemic.

\section{Declarations:}

Funding - The authors received no funding for this study.

Competing interests - The authors declare no competing interest concerning this paper.

\section{References}

Adar, T., Levkovich, I., Castel, O. C. and Karkabi, K. (2017), 'Patient's utilization of primary care: a profile of clinical and administrative reasons for visits in Israel', Journal of Primary Care and Community Health, 8,4, 221-227.

Ayalon, L. (2020), 'There is nothing new under the sun: Ageism and intergenerational tension in the age of the COVID-19 outbreak', International Psychogeriatrics, 1-4.

Bentur, N. and Heymann, A. D. (2020), 'Depressive symptoms and use of health services among older adults in Israel', Israel Journal of Health Policy Research, 9, 1-6.'

Basis, F., Zeidani, H., Hussein, K. and Hareli, S. (2020), 'Drastic reduction in patient visits to the emergency department in a hospital in Israel during the COVID-19 outbreak, compared to the H1N1 2009', International Journal of Health Policy and Management.

Castro-Costa, E., Dewey, M., Stewart, R., Banerjee, S., Huppert, F., Mendonca-Lima, C. ... and Tsolaki, M. (2007), 'Prevalence of depressive symptoms and syndromes in later life in ten European countries: the SHARE study', The British Journal of Psychiatry, 191.5, 393-401.?

DeViva, J. C., Sheerin, C. M., Southwick, S. M., Roy, A. M., Pietrzak, R. H. and Harpaz-Rotem, I. (2016), 'Correlates of VA mental health treatment utilization among OEF/ OIF/OND veterans: Resilience, stigma, social support, personality, and beliefs about treatment', Psychological Trauma: Theory, Research, Practice and Policy, 8,3, 310 .

Elliott, J., Stolee, P., Boscart, V., Giangregorio, L. and Heckman, G. (2018), 'Coordinating care for older adults in primary care settings: understanding the current context', $B M C$ Family Practice, 19,1, 137.

Garcia, S., Albaghdadi, M. S., Meraj, P. M., Schmidt, C., Garberich, R., Jaffer, F. A. ... and Huang, P. P. (2020), 'Reduction in ST-segment elevation cardiac catheterization laboratory activations in the United States during COVID-19 pandemic', Journal of the American College of Cardiology, 75,22, 2871-2872.

Irwin, M., Artin, K.H. and Oxman, M.N. (1999), 'Screening for depression in the older adult: Criterion validity of the 10-item centre for epidemiological studies depression scale (CES-D)', Archives of Internal Medicine, 159, 1701-1704. 
Jahromi, V. K., Mehrolhassani, M. H., Dehnavieh, R. and Anari, H. S. (2017), 'Continuity of care evaluation: The view of patients and professionals about urban family physician program', International Journal of Preventive Medicine, 8.

Kohout, F.J., Berkman, L.F., Evans, D.A., et al. (1993), 'Two shorter forms of the CES-D (Centre for Epidemiological Studies-Depression) depression symptoms index', Journal of Aging and Health, 5, 179-193.

Lazzerini, M., Barbi, E., Apicella, A., Marchetti, F., Cardinale, F. and Trobia, G. (2020), 'Delayed access or provision of care in Italy resulting from fear of COVID-19', The Lancet Child and Adolescent Health, 4,5, e10-e11.

Li, D., Zhang, D. J., Shao, J. J., Qi, X. D. and Tian, L. (2014), 'A meta-analysis of the prevalence of depressive symptoms in Chinese older adults', Archives of Gerontology and Geriatrics, 58,1, 1-9.

Morrow-Howell, N., Galucia, N. and Swinford, E. (2020), 'Recovering from the COVID-19 pandemic: A focus on older adults', Journal of Aging and Social Policy, 1-9.'

Radloff, L.S. (1977), 'The CES-D scale: A self-report depression scale for research in the general population', Applied Psychological Measurement, 1, 358-401.

Radwan, E., Radwan, A. and Radwan, W. (2020), 'Challenges Facing Older Adults during the COVID-19 Outbreak', European Journal of Environment and Public Health, 5,1, em0059?

Rastogi, D., Madhok, N. and Kipperman, S. (2013), 'Caregiver asthma knowledge, aptitude, and practice in high healthcare utilizing children: effect of an educational intervention', Pediatric Allergy, Immunology and Pulmonology, 26,3, 128-139.

Santini, Z. I., Jose, P. E., Cornwell, E. Y., Koyanagi, A., Nielsen, L., Hinrichsen, C. ... and Koushede, V. (2020), 'Social disconnectedness, perceived isolation, and symptoms of depression and anxiety among older Americans (NSHAP): a longitudinal mediation analysis', The Lancet Public Health, 5,1, e62-e70.

Scheier, M.F., Carver, C.S. and Bridges, M.W. (1994), 'Distinguishing optimism from neuroticism (and trait anxiety, self-mastery, and self-esteem): A re-evaluation of the life orientation test', Journal of Personality and Social Psychology, 67, 1063-1078.

Shahid, Z., Kalayanamitra, R., McClafferty, B., Kepko, D., Ramgobin, D., Patel, R. ... and Jones, K. (2020), 'COVID-19 and older adults: what we know', Journal of the American Geriatrics Society, 68,5, 926-929.]

Shinan-Altman, S. and Levkovich, I. (2020), 'COVID-19 precautionary behaviour: The Israeli case in the initial stage of the outbreak'.

Shinan-Altman, S., Levkovich, I. and Tavori, G. (2020), 'Healthcare utilization among breast cancer patients during the COVID-19 outbreak', Palliative and Supportive Care, 1-7.

So, W. K., Chan, S. S., Lee, A. C. and Tiwari, A. F. (2004), 'The knowledge level and precautionary measures taken by older adults during the SARS outbreak in Hong Kong', International Journal of Nursing Studies, 41,8, 901-909.

Tyrrell, C. J. and Williams, K. N. (2020), 'The paradox of social distancing: Implications for older adults in the context of COVID-19', Psychological Trauma: Theory, Research, Practice and Policy, 12,S1, S214.

Wang, S. Y., Chen, L. K., Hsu, S. H. and Wang, S. C. (2012), 'Health care utilization and health outcomes: a population study of Taiwan', Health Policy and Planning, 27,7, 590-599. 
Wang, J., Kong, D., Sun, B. C. and Dong, X. (2020). Health services utilization among Chinese American older adults: Moderation of social support with functional limitation. Journal of Applied Gerontology, 39(5), 481-489.

World Health Organization (WHO). COVID-19: Operational guidance for maintaining essential health services during an outbreak. World Health Organization. (2020). Retrieved from https://www.who.int/publications-detail/covid-19-operational-guidance-for-maintaining-essential-health-services-during-an-outbreak.

Zhang, J.M., Zhang, D. and Chock, T.M. (2014). Effects of HIV/AIDS public service announcements on attitude and behaviour: Interplay of perceived threat and self-efficacy. Social Behaviour and Personality: An International Journal, 42(5), 799-809. 